\title{
Characterization and Cytotoxic Assessment of Ballistic Aerosol Particulates for Tungsten Alloy Penetrators into Steel Target Plates
}

\author{
Brenda I. Machado ${ }^{1, *}$, Lawrence E. Murr ${ }^{1}$, Raquel M. Suro ${ }^{2}$, Sara M. Gaytan ${ }^{1}$, \\ Diana A. Ramirez ${ }^{1}$, Kristine M. Garza ${ }^{2}$ and Brian E. Schuster ${ }^{3}$
}

1 Department of Metallurgical and Materials Engineering, The University of Texas at El Paso, El Paso, TX 79968, USA; E-Mails: lemurr@utep.edu (L.E.M.); smgaytan@miners.utep.edu (S.M.G.); daramirez2@miners.utep.edu (D.A.R.)

2 Department of Biological Sciences, The University of Texas at El Paso, El Paso, TX 79968, USA; E-Mails: rmsuro@miners.utep.edu (R.M.S.); kmgarza@utep.edu (K.M.G.)

3 U.S. Army Research Laboratory, Weapons and Materials Research Directorate (RDRL-WML-H), USA; E-Mail: bschuster@arl.army.mil (B.E.S.)

* Author to whom correspondence should be addressed; E-Mail: bimachado@miners.utep.edu; Tel.: +1-915-630-6404; Fax: +1-915-747-8036.

Received: 10 July 2010; in revised form: 24 August 2010 / Accepted: 25 August 2010 / Published: 26 August 2010

Abstract: The nature and constituents of ballistic aerosol created by kinetic energy penetrator rods of tungsten heavy alloys (W-Fe-Ni and W-Fe-Co) perforating steel target plates was characterized by scanning and transmission electron microscopy. These aerosol regimes, which can occur in closed, armored military vehicle penetration, are of concern for potential health effects, especially as a consequence of being inhaled. In a controlled volume containing 10 equispaced steel target plates, particulates were systematically collected onto special filters. Filter collections were examined by scanning and transmission electron microscopy (SEM and TEM) which included energy-dispersive (X-ray) spectrometry (EDS). Dark-field TEM identified a significant nanoparticle concentration while EDS in the SEM identified the propensity of mass fraction particulates to consist of $\mathrm{Fe}$ and $\mathrm{FeO}$, representing target erosion and formation of an accumulating debris field. Direct exposure of human epithelial cells (A549), a model for lung tissue, to particulates (especially nanoparticulates) collected on individual filters demonstrated 
induction of rapid and global cell death to the extent that production of inflammatory cytokines was entirely inhibited. These observations along with comparisons of a wide range of other nanoparticulate species exhibiting cell death in A549 culture may suggest severe human toxicity potential for inhaled ballistic aerosol, but the complexity of the aerosol (particulate) mix has not yet allowed any particular chemical composition to be identified.

Keywords: tungsten alloys; nanoparticulates; cytotoxic assays; scanning and transmission electron microscopy; aerosol; ballistic penetration

\section{Introduction}

In regards to very small fragments and fragment aerosols, there have been few systematic observations of associated particulate chemistries, size distributions or cytotoxic responses, especially pertinent to respiratory inflammatory responses or more serious respiratory health effect indicators; although recent work by Gold et al. [1] has examined aerosols inside an armored vehicle penetrated by a kinetic energy tungsten heavy alloy (KE WHA) penetrator. In addition, Guillmette et al. [2] have also discussed the health risk for depleted uranium (DU) aerosols. It is now well established that ultra-fine or nanoparticulate materials characteristic of a wide compositional range and particulate morphologies exhibit respiratory inflammatory and cytotoxic effects for a range of human lung cell types [3-10].

The present study is concerned with the systematic collection of aerosol particulates associated with ballistic WHA rod penetration into rolled homogeneous armor (RHA) or related steel armor or armor plate sequences in a containment vessel. This research is also concerned with the characterization of these collected particulates using scanning and transmission electron microscopy; including the analysis of particulate chemistries or elemental compositions utilizing energy dispersive (X-ray) spectrometry (EDS). Finally, filter-collected aerosol particulates were exposed to human epithelial (lung) cells in culture to assess their inflammatory and related respiratory health effects.

\section{Experimental Procedures}

\subsection{Materials and Ballistic Testing}

Sub-scale WHA penetrators were fired into an array of mild steel plates which were encapsulated in a steel containment vessel. The penetrators were hemispherical-nose, $65 \mathrm{~g}$ rods with a length to diameter ratio of 20 . The WHA rods consisted of either $91 \%$ (by weight) $\mathrm{W}, 5.6 \% \mathrm{Ni}, 1.4 \%$ (WA \#1) or $92 \% \mathrm{~W}, 6 \% \mathrm{Ni}, 3 \% \mathrm{Co}$ (WA \#2) or and were fabricated using liquid phase sintering [11]. In Figure 1(a) and (b), we show the typical microstructures of WA \#1 and \#2 (respectively) consisting of pure tungsten particles surround by the matrix phase.

The target array consisted of 10 each of $6.25 \mathrm{~mm}$ thick mild steel plates spaced $12.5 \mathrm{~mm}$ apart backed by a thick RHA block to capture the residual penetrator. The penetrators were fired from a 
$26 \mathrm{~mm}$ smooth bore cannon outfitted with a $37 \mathrm{~mm}$ breach. The launch package consisted of a polypropulux laboratory sabot and obdurator with a steel pusher plate. The penetrators (and entire launch package) were fired at a velocity of $\sim 1.2 \mathrm{~km} / \mathrm{s}$ using approximately $170 \mathrm{~g}$ of type M2 propellant. The approximate composition of the M2 propellant as reported by Roth and Watchtell is shown in Table 1 [12]. In all 12 tests, the penetrators completely perforated all of the thin mild steel plates and were caught in the thick RHA block.

Figure 1. (a) WHA KE penetrator section views. (a) W-Ni-Fe penetrator. (b) W-Ni-Co penetrator. The spherical or near spherical particles in (a) and (b) are $\mathrm{W}$ in the corresponding alloy matrix.
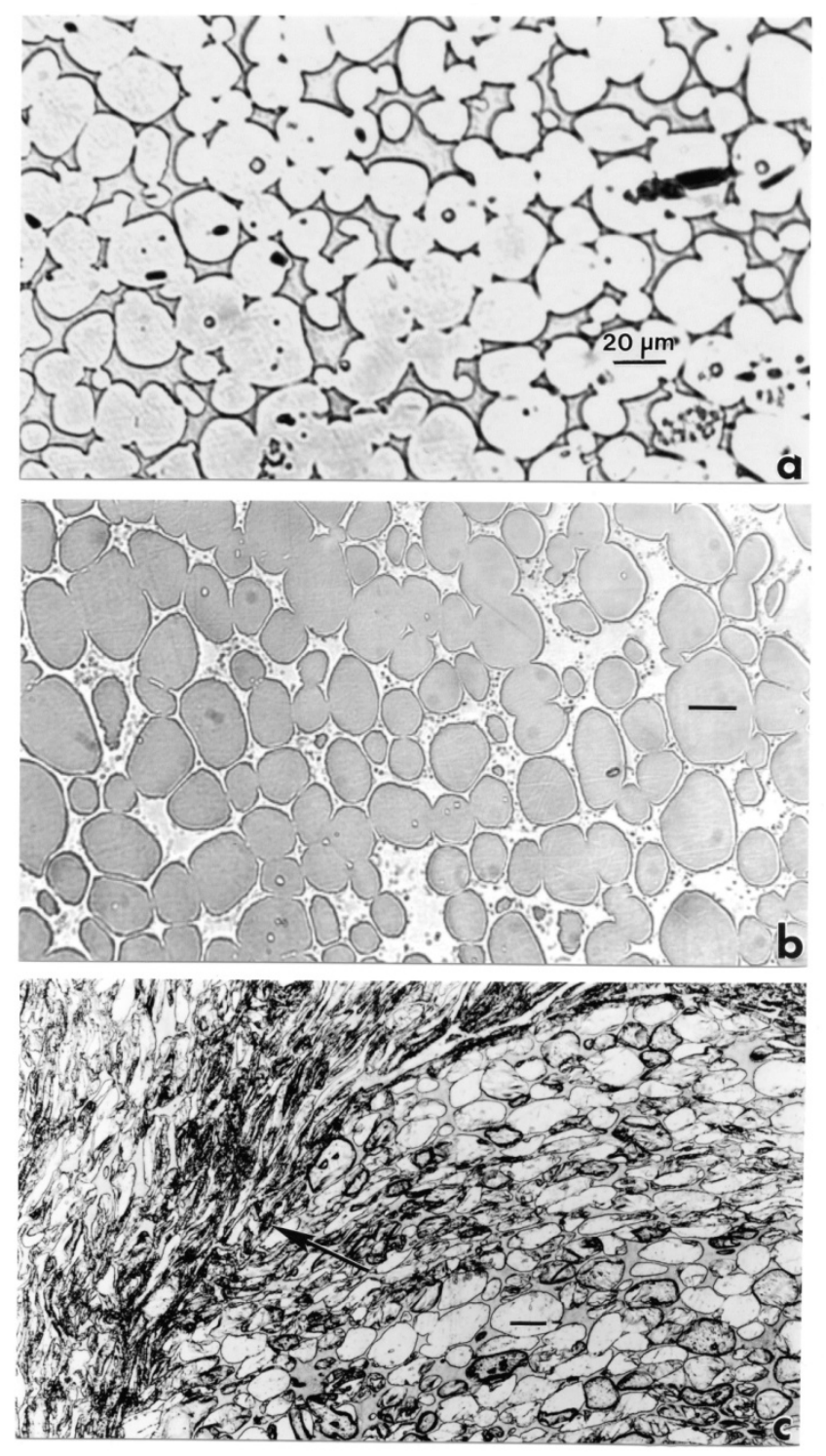

The containment vessel was an RHA cube with a wall thickness of $\sim 25 \mathrm{~mm}$, with an internal open volume that was approximately $60 \mathrm{~cm}$ on a side. This vessel had a removable top which allowed for placement of the target array. The vessel had two ports: a $\sim 10 \mathrm{~cm}$ diameter port through which the WHA penetrators were fired and a smaller $\sim 6 \mathrm{~mm}$ port to allow for aerosol sampling (discussed below). This containment vessel is not a closed system as the larger porter is open during the ballistic 
test and throughout the sampling period. In Figure 2, we show a representative schematic of the ballistic test set-up including the penetrator launch package, mild steel target array and containment vessel.

Table 1. Composition of M2 propellant.

\begin{tabular}{|c|c|}
\hline M2 Composition & \% \\
\hline Nitrocellulose & 77.45 \\
\hline Nitroglycerin & 19.50 \\
\hline $\mathrm{Ba}\left(\mathrm{NO}_{3}\right)_{2}$ & 1.40 \\
\hline $\mathrm{KNO}_{3}$ & 0.75 \\
\hline Ethyl Centralite & 0.60 \\
\hline Graphite & 0.30 \\
\hline
\end{tabular}

Figure 2. Ballistic aerosol particulate generation and collection. The sabot-fired KE rod penetrates 10 mild steel target plates in an RHA cube confinement. A cascade impactor or single-filter collector collects particulates from the debris fields in separate penetration events.

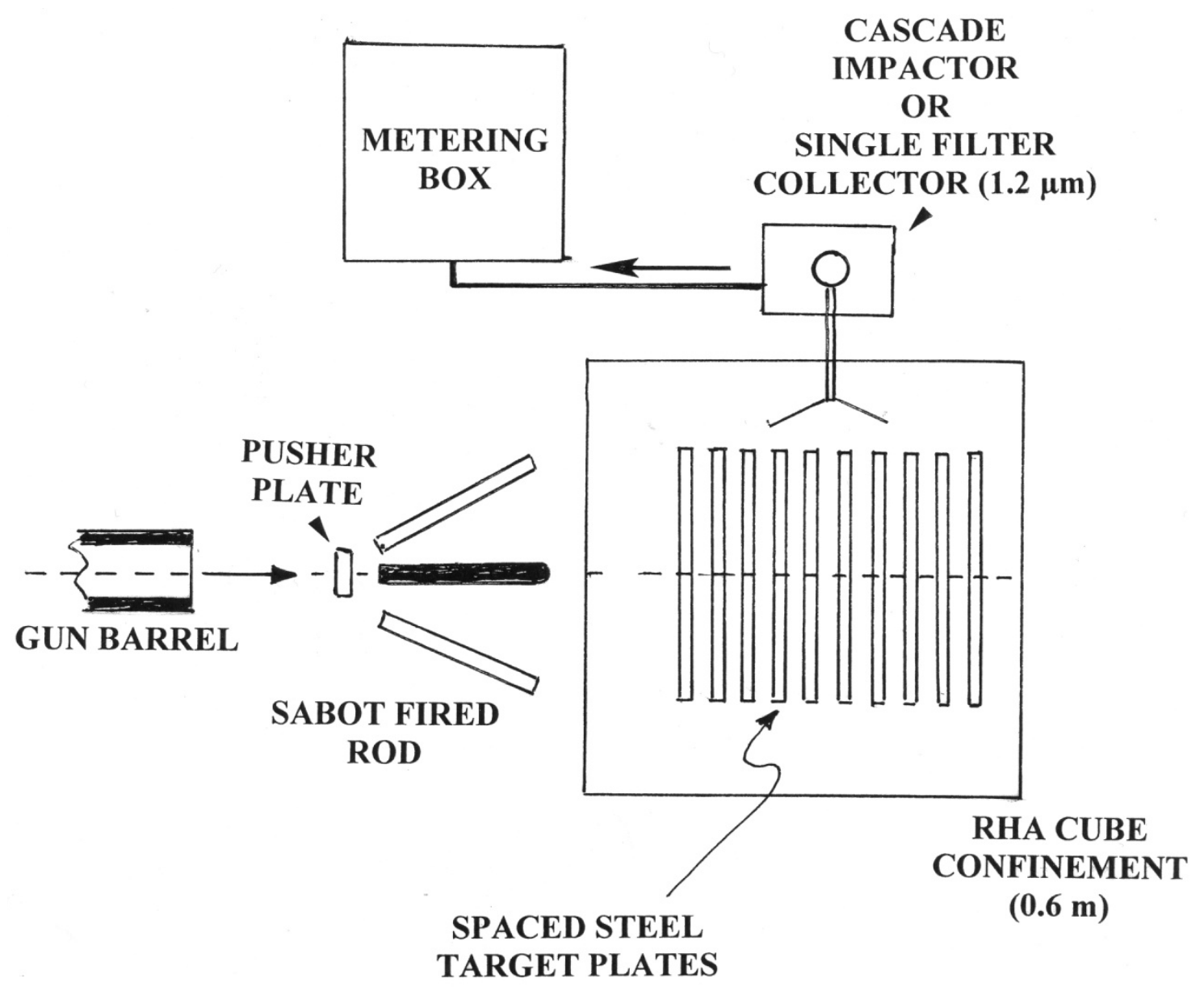




\subsection{Aerosol Collection and Analysis}

Immediately following the ballistic test, aerosol samples were collected using either single-stage filters or by an eight-stage cascade impactor, under non-sterile conditions. The debris (particulate aerosol) generated by the rod impact, penetration and perforation as well as the residual propellant gases were sampled for $\sim 15$ minutes through the respective collection media with an airflow rate of $2 \mathrm{~L} / \mathrm{min}$.

The single stage filters were SKC model 225-321A mixed cellulose ester (MCE) filters with a diameter of $25 \mathrm{~mm}$ and a pore-size of $0.8 \mu \mathrm{m}$. The eight stage cascade impactor utilized Mylar substrates with cut-points ranging from 0.5 to $21 \mu \mathrm{m}$ and with a final backup filter with a $0.25 \mu \mathrm{m}$ pore size. The Mylar substrates were sprayed with Dow Corning 316 silicone release lubricant $\sim 16 \mathrm{~h}$ before initial weighing to provide an adhesive surface for the airborne particles. The total particulate concentrations at each cut point were determined from gravimetric analysis. Each substrate was retained for subsequent analysis in their SKC and Millipore containers and samples for the relative concentrations of $\mathrm{W}, \mathrm{Ni}, \mathrm{Fe}$ and $\mathrm{Co}$. Analysis was conducted following National Institute for Occupational Safety and Health (NIOSH) method 7074 which included cellulose ester filter collection, acid digestion, and analysis by inductively coupled plasma optical emission spectroscopy (ICP-OES). EPA method 200.7 was used for metal analysis.

\subsection{Aerosol Particulate Characterization}

Filter collection samples were halved or quartered and utilized for direct particulate collection observation and analysis by scanning electron microscopy (SEM) and energy-dispersive (X-ray) spectrometry (EDS). The SEM was a field emission SEM (Hitachi S4800) operated at $20 \mathrm{kV}$ accelerating potential to assure adequate excitation potential for EDS. Filter sections were also scraped onto silicon monoxide/formvar-coated 200 mesh, $3 \mathrm{~mm}$ copper grids and a second grid placed on top to form a sandwich for direct observation in the transmission electron microscope (TEM). The single (MCE) filter collections were more suitable for scraping particles onto TEM grids or onto sticky carbon supports for SEM. TEM image analysis was performed either in a Hitachi H-8000 electron microscope operated at $200 \mathrm{kV}$ or a high-resolution TEM (Hitachi H-9500) operating at $300 \mathrm{kV}$ and fitted with a goniometer-tilt stage. Selected-area electron diffraction (SAED) was utilized to observe the degree of crystallinity of the collected particulates and to allow for selective dark-field (DF) imaging utilizing specific diffraction spots. The high-resolution TEM (Hitachi H-9500) operating at $300 \mathrm{kV}$ was also utilized especially for ultra-fine particle (nanoparticle) analysis. It was fitted with an EDAX-EDS system which allowed for nano-probe (point) analysis and scanned-area analysis.

\subsection{In Vitro Cytotoxicity Assays for Particulate Collections}

In this study we utilized direct exposure cytotoxicity assays for filter-collected ballistic particulates as described previously by Soto et al. [8]. Assays were performed for six different assay groups as summarized in Table 2. Direct exposure assays were performed using particulate specimens collected using the single-stage filters (SSF) associated with ballistic impacts of WA \#1 and WA \#2 into the 
steel target array. There was no measurement of particle weight on the filters. Untreated cells (or "media" only cells) served as the negative control; cells exposed to a filter on which nothing had been collected served as the blank control.

Table 2. Direct Contact Cytotoxicity Assay Groups.

\begin{tabular}{|c|c|}
\hline Name & Description \\
\hline SSF-WA \#1 & $\begin{array}{c}\text { Single Stage Filter Collections for } \\
\text { WA \#1 impacting target array }\end{array}$ \\
\hline SSF-WA \#2 & $\begin{array}{c}\text { Single Stage Filter Collections for } \\
\text { WA \#2 impacting target array }\end{array}$ \\
CI6-WA \#1 & $\begin{array}{c}\text { Cascade Impactor Stage 6 } \\
\text { Specimens for WA \#1 impacting } \\
\text { array }\end{array}$ \\
CI6-WA \#2 & $\begin{array}{c}\text { Cascade Impactor Stage 6 } \\
\text { Specimens for WA \#1 impacting } \\
\text { array }\end{array}$ \\
\hline Media & Negative Control-Untreated cells \\
\hline Blank & $\begin{array}{c}\text { Cells exposed to a single stage } \\
\text { filter on which nothing has been } \\
\text { collected }\end{array}$ \\
\hline
\end{tabular}

These assays measure in vitro cell viability or cell death using colorimetric or optical densitometry analysis along with cytokine enzyme linked immunosorbant assay (ELISA) studies which measure the up-regulation and release of interleukins by the cells. The type of ELISA kit used was a BD Biosciences. In recent ELISA studies, we utilized the immortalized A549 human epithelial lung cell line which provides an effective in vitro lung cell model and has been widely adopted as a human lung cell model. The cell line was obtained by the American Tissue Culture Collection (ATCC) from lung carcinomatous tissue from a 58 year old Caucasian male. The A549 cells were cultured in 12-well plates at $0.25 \times 10^{6}$ cells per well with F-12 Ham's media supplemented with $10 \%$ Fetal Bovine Serum (FBS) and 5\% penicillin/streptomycin (PS) for several hours to allow the cells to adhere. The cells were then exposed for $48 \mathrm{hs}$ (standard time for acute exposures) with $1 / 4$ of the indicated filter with the collection side facing towards the monolayer of cells. Following the exposure period, the filters were removed; the cells were harvested, and were then transferred into a 96-well flat bottom plate to assess viability via the MTS Assay. This colorimetric assay assesses relative viability as a function of color, which is directly proportional to the amount of cells available to convert the substrate into a color product. CellTiter 96 Aqueous One Solution Reagent (Promega), which contains the tetrazolium compound 3-(4, 5-dimethylthiazol-2-yl)-5-(3-carboxymethoxyphenyl)-2-(4-sulfophenyl)$2 \mathrm{H}$-tetrazolium, inner salt (MTS), was added to each well. The plate was then incubated for $2 \mathrm{~h}$ at 37 ${ }^{\circ} \mathrm{C}$ in a humidified, $5 \% \mathrm{CO}_{2}$ atmosphere. Finally, the absorbance was recorded at $490 \mathrm{~nm}$ using a 96well plate spectrophotometer reader. The plate was a VersaMax Tunable Microplate Reader of Molecular Devices. Relative viability results were obtained by 1way ANOVA with Bonferroni's Multiple Comparison Test. 
Interleukin (IL)-6 and IL-8 secretion by A549 cells was measured using a commercial human IL-6 and IL-8 enzyme-linked immunosorbent assay (ELISA) kit (Biosource Human IL-6/IL-8 CytoSet). Supernatants were obtained $48 \mathrm{hs}$ after exposure to the different filters and were stored at $-20{ }^{\circ} \mathrm{C}$ until subjected to ELISA analysis following the manufacturer's protocol. The ELISA plates were coated 12 to $18 \mathrm{~h}$ at $4{ }^{\circ} \mathrm{C}$ with the capture antibody (Anti-Human IL-6 or IL-8). The plates were then blocked at room temperature with assay buffer for $1 \mathrm{~h}$ to prevent non-specific antigen binding. Next, the standards (Recombinant Human IL-6 or IL-8) and samples were added in duplicate (2 wells per treatment), immediately followed by the addition of the working detection antibody (Anti-Human IL-6 or IL-8 Biotin) and incubated for $2 \mathrm{~h}$ at room temperature. Subsequently, the plates were washed and the working streptavidin-horseradish peroxidase (HRP) solution was added to each well for 30 min. The enzyme substrate solution tetramethylbenzidine (TMB) was added for color development and finally, the enzyme reaction was stopped by the addition of the stop solution containing hydrosulfuric acid $\left(\mathrm{H}_{2} \mathrm{SO}_{4}\right)$. Absorbance was measured at $450 \mathrm{~nm}$ using a 96-well plate spectrophotometer reader.

\section{Results and Discussion}

\subsection{Aerosol Particulate Size Distribution and Composition}

In Tables 3 and 4, we should the typical sampling data associated with particulate specimens captured using the cascade impactors for the WHA rod impact regime. As noted previously, these specimens were sampled to determine the relative fractions of $\mathrm{W}, \mathrm{Ni}, \mathrm{Fe}$ and Co using ICP-OES while the total particulate weights (and concentrations) were determined using gravimetric methods. The "Total Particulate Concentrations" presumably include a large fraction of undetermined particulate including organic compounds and residual compounds resulting from combustion of the M2 propellant

Table 3. Particulate collection for WA \#1 impact experiment.

\begin{tabular}{|c|c|c|c|c|c|c|}
\hline Stage & $\begin{array}{l}\text { Cut Point } \\
\text { (microns) }\end{array}$ & $\begin{array}{c}\text { Total } \\
\text { Particulate } \\
\text { Weight } \\
\text { (mg) } \\
\end{array}$ & $\begin{array}{c}\text { Total } \\
\text { Particulate } \\
\text { Concentration } \\
\left(\mathbf{m g} / \mathrm{m}^{3}\right)\end{array}$ & $\begin{array}{c}\text { Iron Conc. } \\
\left(\mathbf{m g} / \mathbf{m}^{3}\right)\end{array}$ & $\begin{array}{c}\text { Nickel } \\
\text { Conc. } \\
\left(\mathbf{m g} / \mathbf{m}^{3}\right)\end{array}$ & $\begin{array}{c}\text { Tungsten } \\
\text { Conc. } \\
\left(\mathrm{mg} / \mathrm{m}^{3}\right)\end{array}$ \\
\hline 1 & 21 & 0.432 & 17 & 14.8 & 0.286 & 1.92 \\
\hline 2 & 15 & 0.052 & 2 & 4.51 & 0.134 & 1.36 \\
\hline 3 & 10 & 0.000 & 0 & 3.52 & $\mathrm{ND}$ & 0.217 \\
\hline 4 & 6 & 0.049 & 2 & 7.31 & 0.118 & 0.318 \\
\hline 5 & 3.5 & 0.798 & 31 & 21.0 & 0.312 & 0.736 \\
\hline 6 & 2 & 0.953 & 38 & 20.9 & 0.290 & 0.781 \\
\hline 7 & 0.9 & 0.677 & 27 & 9.97 & 0.142 & 0.625 \\
\hline 8 & 0.5 & 0.204 & 8 & 2.25 & ND & 0.401 \\
\hline $\begin{array}{c}\text { Backup } \\
\text { Filter }\end{array}$ & 0.25 & 0.878 & 35 & 4.89 & ND & 2.59 \\
\hline Total & & & 160 & 89.2 & 1.28 & 8.95 \\
\hline Respirable & & & 106 & 65.0 & 0.862 & 3.08 \\
\hline
\end{tabular}

ND-Concentration below instrument detection limit. 
At all particulate sizes, Fe is the predominant constituent of all the metals samples. Throughout the respirable zone (including stages 3 through 8 of the cascade impactor), Fe-containing particulates are a factor of roughly 25 times the combine $\mathrm{Ni}$ and $\mathrm{W}$ concentrations. Analysis of the backup filter shows a smaller relative ratio of $\mathrm{Fe}$ to $\mathrm{W}$, however the metallic constituents make up a much smaller relative fraction; for example in table 3, the total particulate concentration of $35 \mathrm{mg} / \mathrm{m}^{3}$ includes only $\sim 7.5 \mathrm{mg} / \mathrm{m}^{3}$ from $\mathrm{W}, \mathrm{Ni}$ and $\mathrm{Fe}$.

Table 4. Particle collection for $91 \% \mathrm{~W}-6 \% \mathrm{Ni}, 3 \%$ Co (WHA) penetrator impact experiment.

\begin{tabular}{|c|c|c|c|c|c|c|c|}
\hline Stage & $\begin{array}{c}\text { Cut } \\
\text { Point } \\
(\mu \mathrm{m})\end{array}$ & $\begin{array}{c}\text { Total } \\
\text { Particulate } \\
\text { Weight } \\
\text { (mg) }\end{array}$ & $\begin{array}{c}\text { Total } \\
\text { Particulate } \\
\text { Concentration } \\
\left(\mathbf{m g} / \mathbf{m}^{3}\right)\end{array}$ & $\begin{array}{c}\text { Cobalt } \\
\text { Conc. } \\
\left(\mathbf{m g} / \mathbf{m}^{3}\right)\end{array}$ & $\begin{array}{c}\text { Iron } \\
\text { Conc. } \\
\left(\mathbf{m g} / \mathbf{m}^{3}\right)\end{array}$ & $\begin{array}{c}\text { Nickel } \\
\text { Conc. } \\
\left(\mathbf{m g} / \mathbf{m}^{3}\right)\end{array}$ & $\begin{array}{c}\text { Tungsten } \\
\text { Conc. } \\
\left(\mathbf{m g} / \mathbf{m}^{3}\right)\end{array}$ \\
\hline 1 & 21 & 0.216 & 6 & ND & 5.85 & 0.114 & 0.274 \\
\hline 2 & 15 & 0.000 & 0 & ND & 1.70 & ND & 0.102 \\
\hline 3 & 10 & 0.254 & 7 & ND & 1.87 & ND & 0.106 \\
\hline 4 & 6 & 0.030 & 1 & ND & 3.96 & 0.076 & 0.185 \\
\hline 5 & 3.5 & 0.841 & 22 & 0.088 & 13.70 & 0.208 & 0.535 \\
\hline 6 & 2 & 0.981 & 26 & 0.068 & 12.40 & 0.174 & 0.580 \\
\hline 7 & 0.9 & 0.592 & 16 & $0.184^{*}$ & 5.17 & $0.079 *$ & $0.125 *$ \\
\hline 8 & 0.5 & 0.310 & 8 & 0.075 & 3.07 & ND & 0.417 \\
\hline $\begin{array}{c}\text { Backup } \\
\text { Filter }\end{array}$ & 0.25 & 1.131 & 30 & ND & 4.20 & 0.090 & 1.380 \\
\hline Total & & & 116 & 0.415 & 51.90 & 0.741 & 3.700 \\
\hline Respirable & & & 80 & 0.415 & 40.20 & 0.537 & 1.950 \\
\hline
\end{tabular}

ND-Concentration below instrument detection limit.

\subsection{Particulate Characterization by SEM and TEM}

Figure 3 shows typical stage $6(\sim 2 \mu \mathrm{m}$ cut point) particulate (cascade impactor) collections observed directly in the SEM. Figure 3(a) illustrates particle aggregates for WA \#1 projectile firing. Consistent with the particle analyses shown in Table 3, the dominant element shown in the EDS insert is Fe, with some observable $\mathrm{W}$. There is no detectable Ni. The Fe found in the SEM/EDS analysis includes contributions from both the WHA matrix as well as from the mild steel target array; the latter is believed to provide the most significant contribution. It is not possible to separate the contribution from Fe found originally in the WHA from that in the target array. It was also observed that most of the spherical particles, ranging from 1 to $2 \mu \mathrm{m}$ in Figure 3(a), were Fe. Figure 3(b) illustrates, similar particle aggregates for a penetration rod of WA \#2 corresponding to Table 4. Similar to Figure 3(a), the EDS insert illustrates a propensity of target $\mathrm{Fe}$, and there is no Ni or Co detected. 
Figure 3. Stage 6 (Tables 1 and 2) cascade impactor filter samples observed in the FESEM. (a) WA \#1 WHA penetrator event. (b) WA \#2 WHA penetrator event. The corresponding energy-dispersive X-ray spectra (EDS) are inserted.
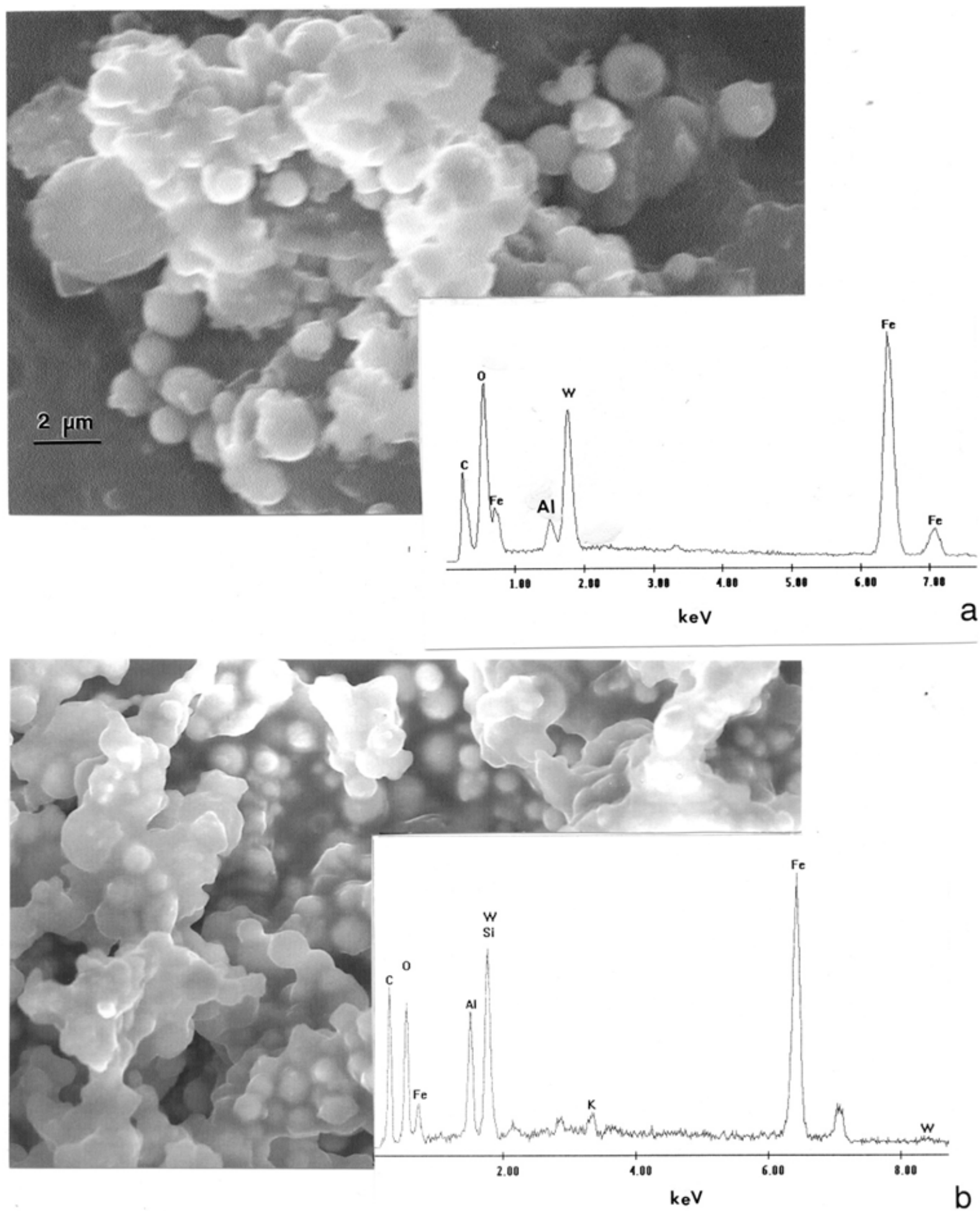
Figure 4 illustrates an aggregate of particles observed for a typical MCE (single-filter) collection in the SEM. The propensity of particles ranging from 0.5 to $2 \mu \mathrm{m}$ are spherical Fe particles. The EDS illustrates a similar composition (as found throughout) dominated by Fe, with some W. The EDS insert in Figure 4 also shows significant oxygen suggesting that many particles may be oxides: $\mathrm{Fe}-\mathrm{O}\left(\mathrm{Fe}_{2} \mathrm{O}_{3}\right)$ as suggested in a previous study by Gold [13].

Figure 4. Single filter sample observed with the FESEM for a W-NiFe WHA penetrator event. The corresponding energy-dispersive X-ray spectrum for the large, spherical iron particle (arrow) is included.

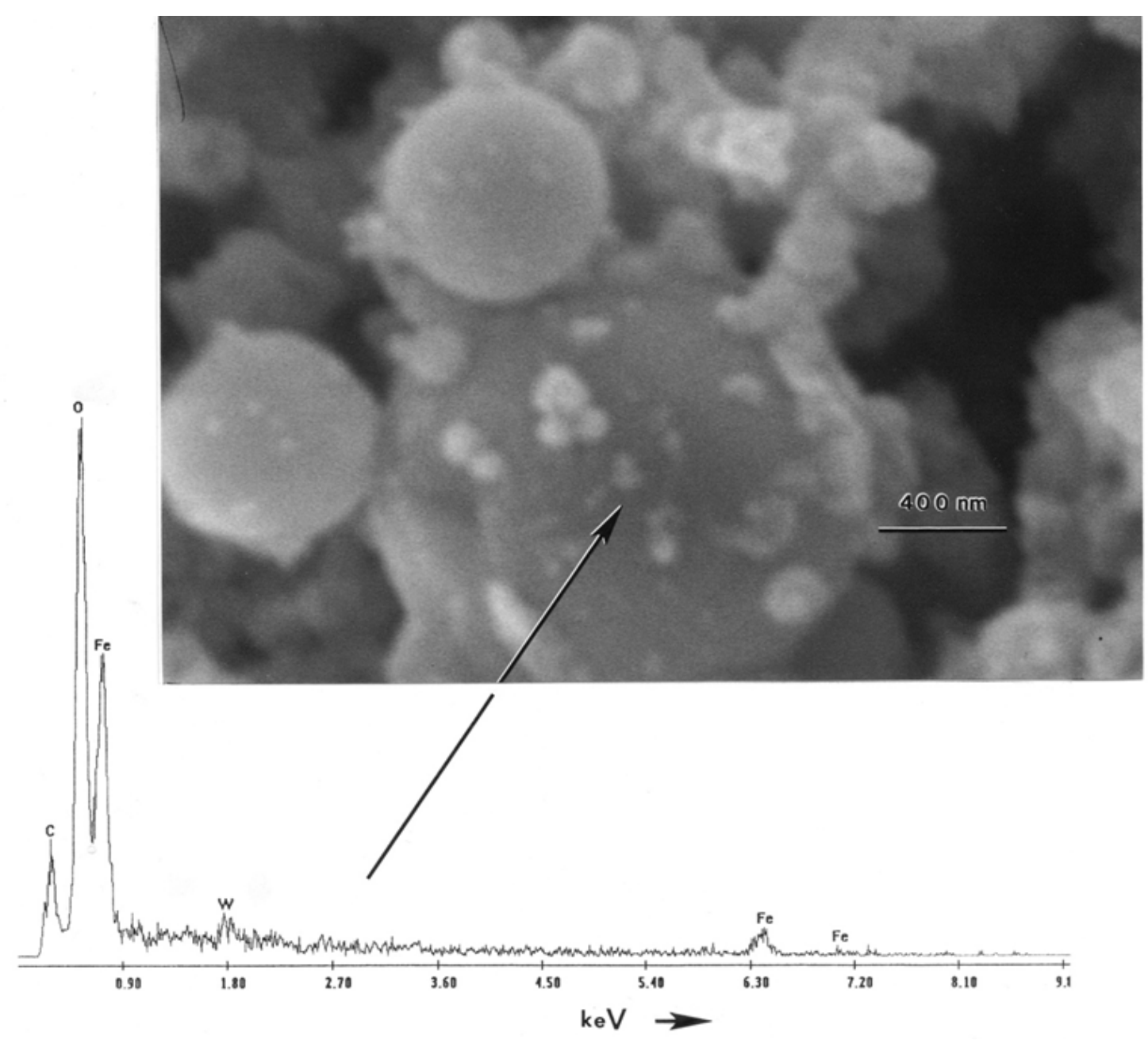

Figure 5 shows for comparison single (MCE) filter particulate collections for both WA \#1. This SEM image illustrates notable clarity, in contrast to Figure 4 because the filters were pressed against sticky carbon SEM tabs which removed the particles and placed them on an electrically conducting support which largely eliminated charging of the specimens. The EDS insert in Figure 5 is essentially the same as those shown in Figures 3 and 4, with small variations in $\mathrm{W}$ concentrations and no Ni spectra. 
Figure 5. Single-filter sample particulates scraped from the filters into conductive carbon tape and observed in the FESEM. WA \#1 WHA rod penetrator event image with broad-area EDS.

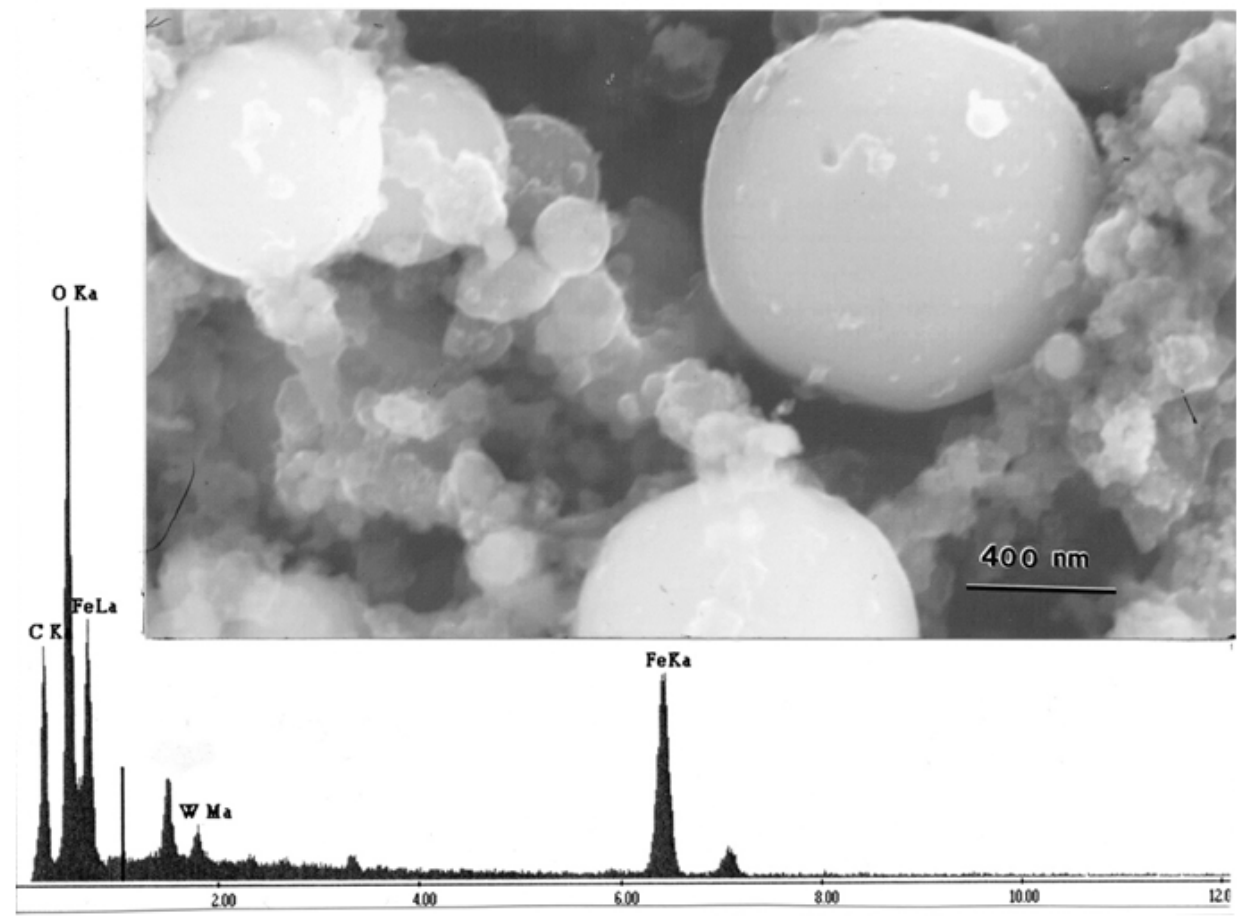

Figure 6 shows an analytical sequence for TEM observations of a small aggregate of particulates scrapped from an MCE filter for a WA \#1 penetration event. Figure 6(a) illustrates a bright-field image of the aggregate while Figure 6(b) shows the corresponding selected-area electron diffraction (SAED) pattern. Only a few diffraction spots are observable above the diffuse background and the arrow indicates a specific diffraction spot for one of the larger (Fe) particles in Figure 6(a). This particle is shown magnified in Figure 6(c) while Figure 6(d) illustrates the corresponding dark-field image of Figure 6(c) utilizing the specific diffracting spot at the arrow in Figure 6(b). It can be noted that this particle is a bi-crystal with some microstructure visible in the left-hand crystal (bright). Several other much smaller (nano) crystal fragments are indicated at arrows in Figure 6(d). The smallest observable dark-field particle images in Figure 6(d) are observed to be $\sim 5 \mathrm{~nm}$.

Figure 7 provides additional TEM bright-and dark-field image sequences showing the very small nanoparticulates to range from $\sim 10$ to $50 \mathrm{~nm}$. Numerous nanoparticulate aggregates are also visible in the dark-field images of Figures 7(b) for WA \#1 and W-Ni-Co projectile firings, respectively. The SAED pattern inserts in Figures 7(a) show prominent arrays of diffraction spots from various crystalline particles, there is some diffuse diffraction which is less prominent than the SAED pattern insert in Figure 6(b). However this diffuse diffraction along with careful perusal of the bright-field images of particulates in Figures 6(a) and 7(a), suggests some organic (carbon) component of the aggregates, which is also reflected in the carbon peaks in all EDS inserts (Figures 3-5). At least some of this organic debris represents residual combustion material associated with the gun firing of the rod projectiles as noted above. 
Figure 6. WA \#1 WHA penetrator event aerosol particulates scraped from a single filter collector onto a grid sandwich and observed in the TEM. (a) Bright-field image of particulate aggregates. (b) Selected-area electron diffraction (SAED) pattern for aggregates in (a). Magnified bright-field image of (a). (d) Dark-field image of (c) using diffraction spot at arrow in (b). Nanoparticle images are indicated by the arrow. The magnification markers are designated by the marker in (a). The accelerating potential was $300 \mathrm{kV}$.
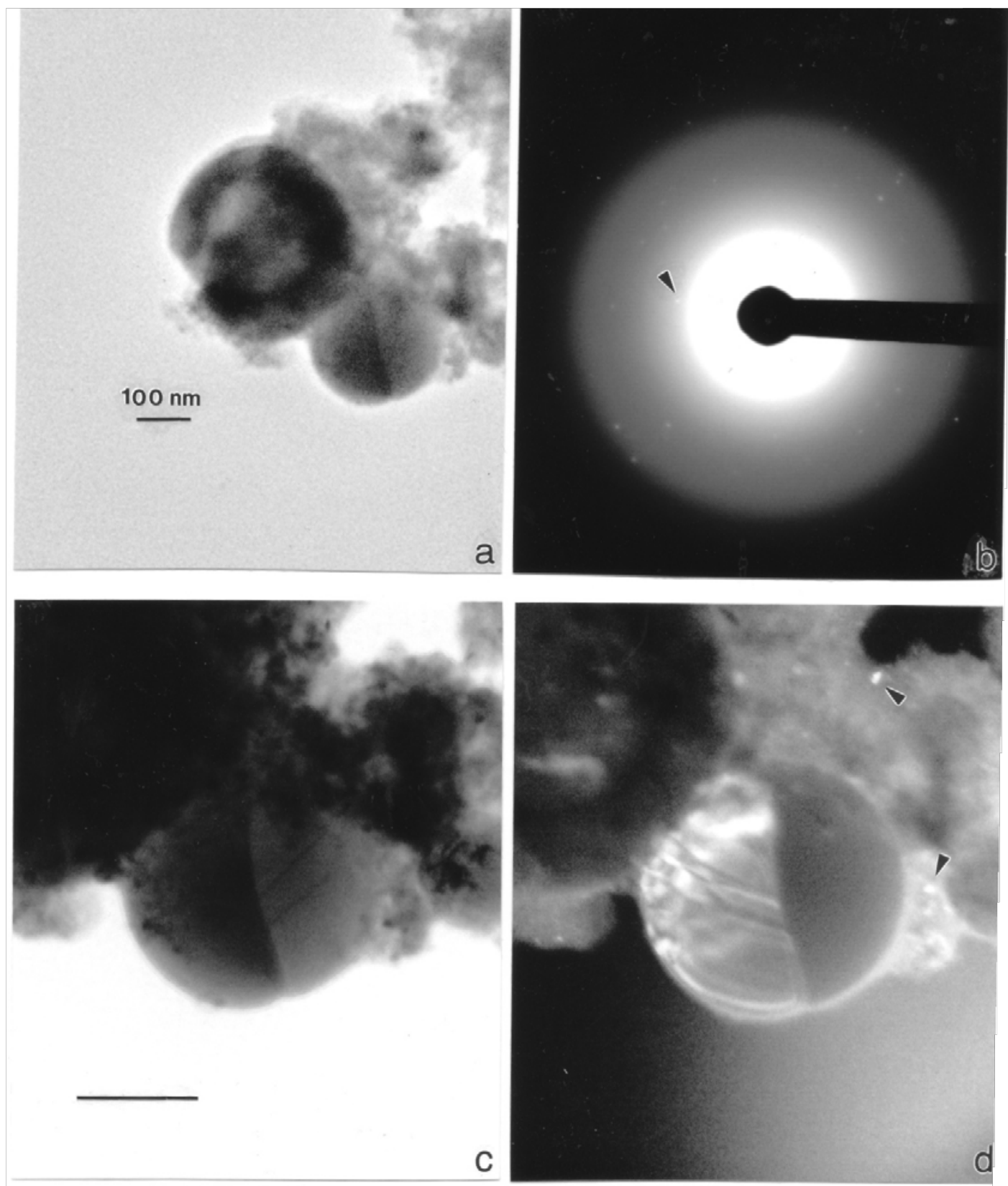
Figure 7. Single-filter collection sample for a WA \#1 WHA rod penetration event scraped onto a grid sandwich and observed in the TEM. (a) Bright-field image of nanoparticulate aggregates. The SAED pattern insert illustrates broad crystallinity. (b) Dark-field image of (a) using electron diffraction spots with the aperture area shown circled in SAED pattern insert in (a). Nanocrystalline particulates are designated typically by arrows. (200 kV accelerating potential).
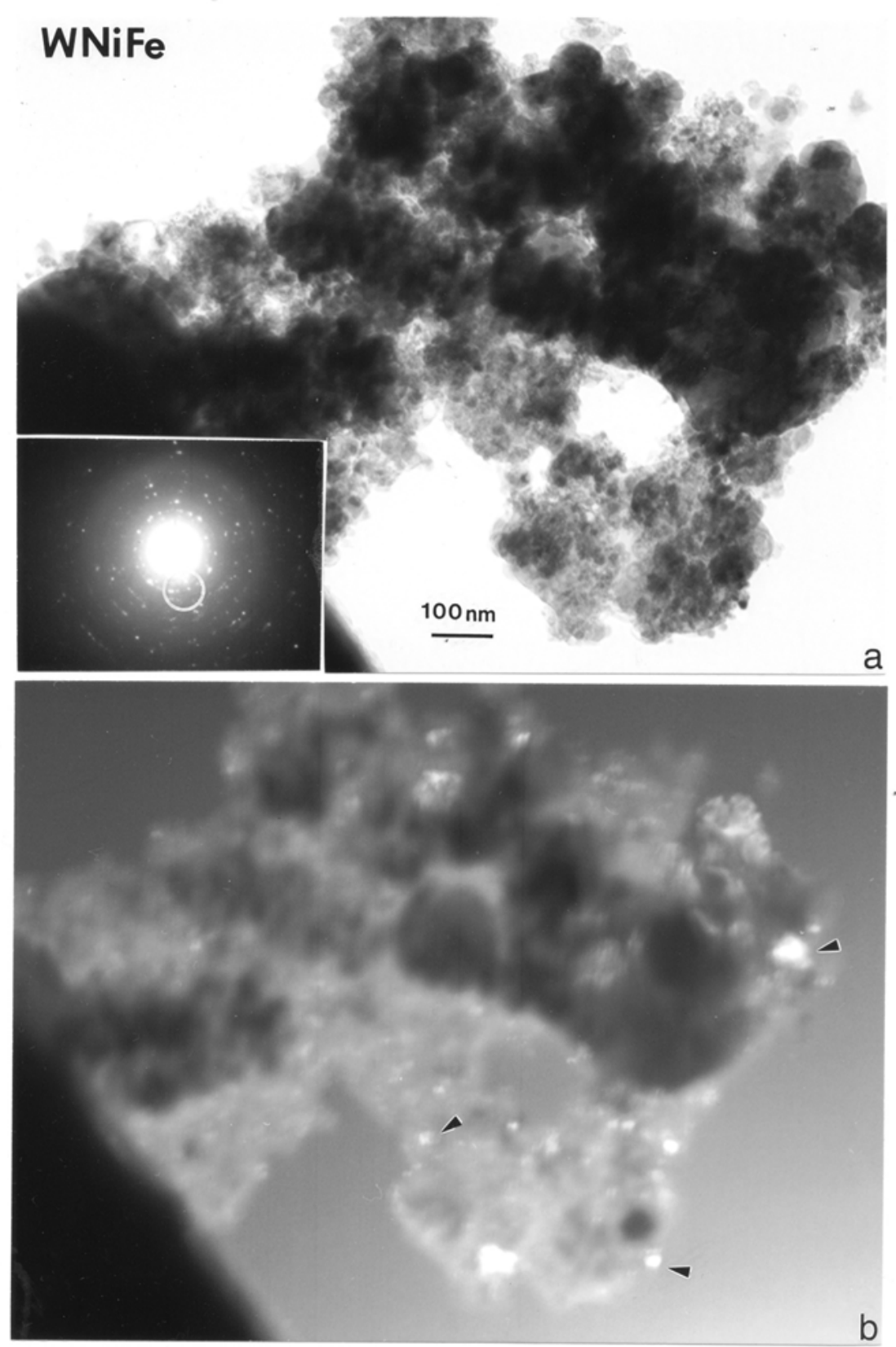

\subsection{Cytotoxicity Assays}

Figure 8 shows the in vitro assays in A549 cell cultures for $48 \mathrm{~h}$ for the six assay groups (see Table 2). Each experiment was done in duplicate ( 2 wells per treatment for each experiment) and each experiment was done twice. No significant reduction in A549 cell viability was associated with direct exposure to the stage 6 impactor collection (see Table 2, CI6-WA \#1 and CI6-WA \#2). In contrast, 
direct exposure of single stage filter samples from impacts of both WA \#1 and 2 (SSF-WA \#1 and SSF-WA \#2) show very significant or highly cytotoxic responses. Respiratory epithelial cells such as the A549 lung model cells used in these assays have the ability to synthesize and release inflammatory cytokines such as interluken (IL)-6 and IL-8, as well as growth factors that modulate differentiation and inflammatory cells. Interleukin 6 (IL-6) and IL-8 are considered markers for inflammatory response both in the A549 cell culture models and in bronchial alveolar lavage fluids in animals [14-19], ideally providing a biomarker link between in vitro and in-vivo studies. Specifically, IL-6 has been associated with allergic responses involving asthma [16-19] while IL-8 has been linked with chronic obstructive pulmonary disease (COPD) [5,18-20]. The cells are killed so rapidly that there is no time to respond, as evident in the complete lack of IL production in Figure 8(d) and (e). The cells were killed so rapidly that only aggregates of particulates uptaken by the cells remained.

Figure 8. Comparative cytotoxicities (as relative A549 epithelial cell viability in filter-collected samples) and cytokine production. (a) and (b) Single filter exposure cell culture assays compared to media or untreated cell control and blank filter control for $48 \mathrm{~h}$ exposure. Specific ballistic event numbers are shown for WA \#1 and WA \#2 penetration events. (c) Cascade impact stage 6 cell culture assays which show no effect. (d) and (e) show cytokine (IL 6 and IL 8) assays. Data is presented as the mean \pm SEM of duplicate samples and is one of two representative experiments $(* p \leq 0.01)$.
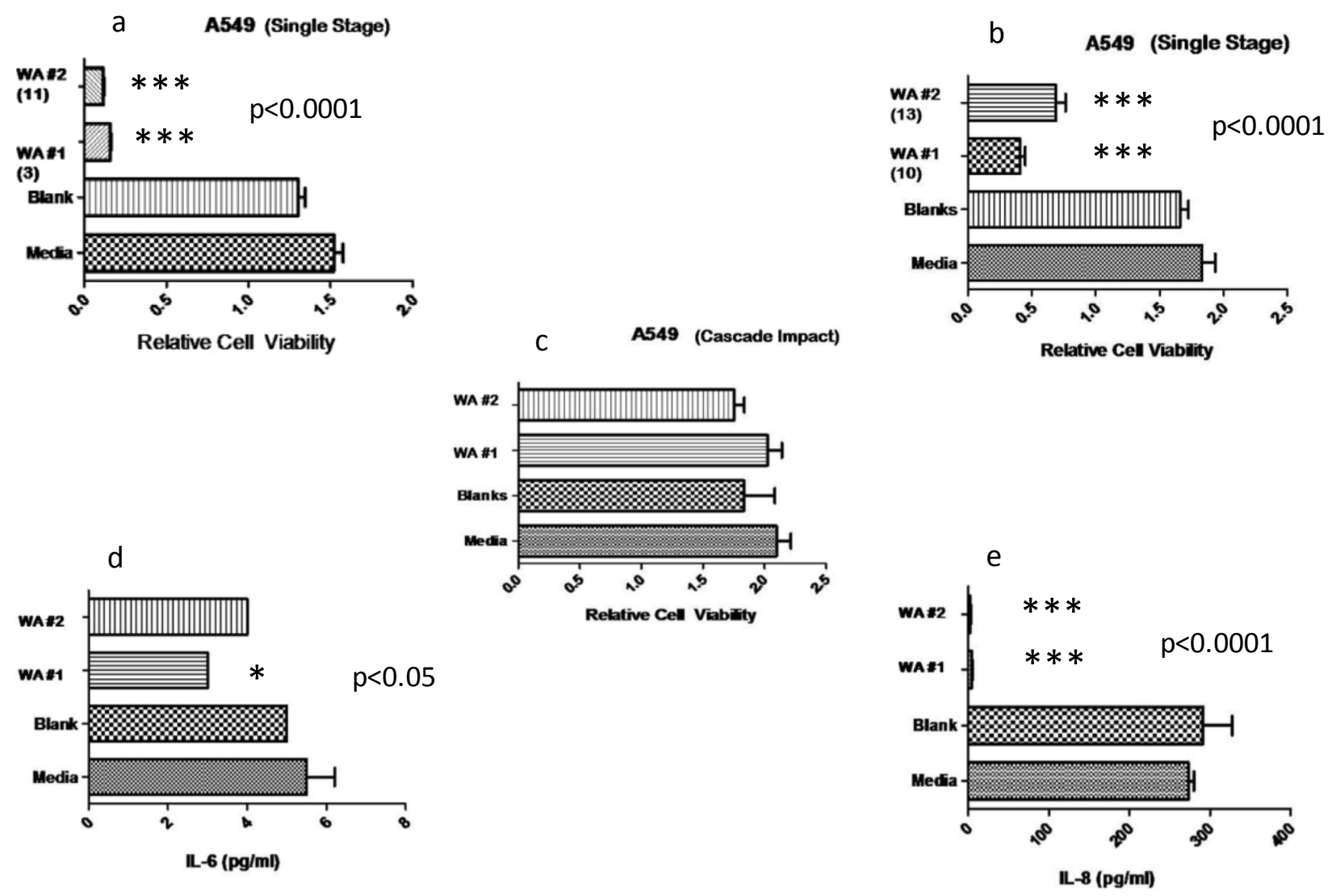


\subsection{Comparative Cytoxicity of These and Other Nanoparticulate Species}

Specimens collected on the single-stage filters represent the full spectrum aerosol specimens generated and collected. The stage 6 cascade impactor collections represent a subset of that present in the single-stage collections. Ideally, the latter collections contained a smaller overall size fraction of particulates, but both the single filter collections as well as the stage 6 impactor collections contained nanoparticulates and nanoparticulate aggregates as illustrated in figures 3-5. However, in contrast to the single filter cytotoxicities illustrated in Figure 8(a) and (b), there was no significant reduction in A549 cell viability for the stage impactor assays (CI6-WA \#1 and \#2) (Figure 8(c)). This indicates a sharp delineation in cytotoxicity with particulate and especially 6 nanoparticulate concentrations since, as indicated in Tables 1 and 2, the total and respirable fractions are roughly 4 times greater than the particulate concentrations on the stage 6 impactor filters, even though these filters contained significantly more particulates (particulate concentrations) than other impactor filters (Tables 1 and 2).

In contrast to other filter exposure cytotoxicity assays and standard in vitro cytotoxicity comparisons for a wide range of other nanoparticulate materials in culture (A549 cell exposure), the ballistic aerosol particulates are highly toxic [8,21]. Similar features have been observed for a wide range of nanoparticulates and nanoparticulate aggregates which also exhibit significant cytotoxicity [6].

\subsection{Respiratory Effects of Nanoparticulates}

There is a plethora of evidence over at least the past two decades that metal and metal oxide particles, especially nanoparticles are toxic, especially in the pulmonary system. Publications by Cugell et al. [22]; Sullivan et al. [23] and Buzea et al. [24] to name only a few attest to the health effects of $\mathrm{Co}, \mathrm{Ni}, \mathrm{W}$, and $\mathrm{Fe}$. These exposures to $\mathrm{Co}, \mathrm{Ni}$, and $\mathrm{W}$ can cause pulmonary fibrosis asthma, pulmonary eodema and pneumonia among other effects. Nickel and its compounds in particular have been demonstrated to cause nasal and lung cancers in the longer term for repeated exposure [23,24]. In addition, a wide range of metal oxides have also been demonstrated to be toxic or cytotoxic, especially iron oxide [24,25] as illustrated in the comparative cytotoxicity data in Figure 6.

Inhaled particulates (including coarse nanoparticles $<1 \mu \mathrm{m}$ ) initially encounter the mucocillary clearance by cilia of the bronchial epithelial cells which moves the larger particles $(>1 \mu \mathrm{m})$ towards the upper respiratory tract. However the truly nanoparticles $(<1 \mu \mathrm{m})$ migrate to the alveoli where phagocytes and other cells with phagocytic abilities work to arrest them. However with phagocytic impairment, or for nanoparticles $<100 \mathrm{~nm}$ which are not readily phagocitized, nanoparticles can accumulate and even aggregate to create oxidative stress and inflammation [26]. This can lead to various diseases while inflammation plays a major role in coronary heart disease and airway diseases such as asthma and chronic obstructive pulmonary disease (COPD). Severe inflammation is also associated with the onset of autoimmune disease. A prominent mechanism responsible for the variety of nanoparticle toxicities assumes a shift in the redox balance of the cells towards oxidation as a consequence of the formation of reactive oxygen species (ROS) which can, in the longer term, lead to DNA damage [24]. In addition to oxidative stress, some nanoparticles can enhance the expression of specific viral receptors and lead to severe inflammation when exposed to viral infections while other 
nanoparticles can decrease the expression of certain viral and bacterial receptors which lowers the resistance to some types of micro-organisms [27]. This phenomenon has been described previously for the in vitro assays for the collected aerosol particles on filters where IL-8 inhibition prevents the A549 epithelial cells from normal function. This would prevent the lungs from mounting an effective response to inhaled microbes.

Oberdörster, et al. [26] have demonstrated that aggregated nanoparticles are not as toxic as smaller concentrations of single particles. However, considerable aggregation observed in this study would suggest that in lung fluid, many of the aggregated particles, particularly those $<100 \mathrm{~nm}$, would disaggregate. Moreover, Murr et al. [25] have shown that a wide range of aggregated nanoparticulates are noticeably cytotoxic. Additionally high concentrations of aggregated nanoparticles can produce lung burden and pulmonary tissue damage depending upon the lung clearance rate [24]. Smaller nanoparticles $(<30 \mathrm{~nm})$ can emulate virus diameters and cross physiological barriers, entering the circulatory and lymphatic systems where they can translocate to various organs [28]. Inhaled nanoparticles $<100 \mathrm{~nm}$ have also been shown to reach the brain via olfactory nerves as well as the blood-brain barriers [24,30]. Studies have suggested that high concentrations of metals such as $\mathrm{Cu}, \mathrm{Al}$, $\mathrm{Fe}$, and others, together with oxidative stress, may initiate and even promote neurodegenerative diseases such as Parkinson's and Alzheimer's diseases [24].

Current research reviews indicate that with few exceptions, nanoparticles and nanoparticle aggregates are toxic (if not cytotoxic) to living organisms [24,25]. However, the relationship between nanoparticle exposure and immune response is not well known. The degree of toxicity for specific nanoparticles cannot be extrapolated from bulk properties, and materials which are nontoxic in bulk form may be toxic in nanoparticle or nanoparticle aggregate form [24].

\section{Conclusions}

As in the case for environmental particulate regimes, including the outdoor environment, nanoparticulates accounted for the most significant number concentration or particulate abundance of the ballistic aerosol, with mass abundances exceeding $150 \mathrm{mg} / \mathrm{m}^{3}$ (Table 3). This might be compared to mass concentrations of $\sim 90 \mathrm{mg} / \mathrm{m}^{3}$ for burning tire soot or natural gas combustion particulate mass concentrations of $\sim 0.01 \mathrm{mg} / \mathrm{m}^{3}$ as illustrated in the comparative cytotoxicity data in Figure 8(e) [30]. Correspondingly, these small mass abundance values represented the most cytotoxic aerosol category in contrast to larger concentrations of other soot species. In this work, ballistic aerosol composed largely of nanoparticulates averaging $\sim 10 \mathrm{~nm}$ (Figures 6-7) was shown to be so highly toxic to human epithelial cells over a short time $(48 \mathrm{~h})$ in culture that the cells died (Figure 8(c) and (d)) before they could produce any responsive cytokines (IL-6 or IL-8) (Figure 13(c) and (d)). Lower ballistic aerosol concentrations measured to be roughly $1 / 4$ of the topic concentrations showed no measurable cell (A549 human epithelial) death after $48 \mathrm{~h}$ in culture. While the concentration cut off and exposure time in culture to affect significant epithelial cell death was not determined, the aerosol characterization and cytotoxicity assays provide compelling evidence for significant health hazards "for exposure, inhalation, and respiration of aerosolized metals" as earlier suggested by Gold et al. [1] for military crew compartment armor steel perforation by a WHA-KE penetrator of the type studied in this work. 
Although the nanoparticulate species or stoichiometries contributing significantly to epithelial cell death (Figures 8(a), (b), (c), and (d)) are not fully characterized, considerable quantities (concentrations) of iron oxides and other metal or metal oxide nanoparticles contribute significantly to the ballistic aerosol and these have already been demonstrated to be highly cytotoxic as illustrated in Figure 8(f). It is also not known how these various nanoparticle species contribute synergistically to the highly toxic behavior of the ballistic aerosol. Despite these shortcomings, this research suggests that there is the potential for severe or chronic respiratory health effects associated with even $\mathrm{s}$ hort-term exposure to ballistic aerosol at high concentrations associated with KE penetrator perforation of crew compartments in a variety of armored vehicles.

\section{Acknowledgements}

This research was supported by the U.S. Army Research Laboratory (ARL), Aberdeen Proving Ground, MD (under Contract No. W9119X-08-D0001, Amend. \#0002); the NIH-funded RCMI Grant \#2G12RR008124. R.M.S. is supported by a RISE Graduate Student Fellowship Grant \#R25GM069621-02. The two cores used for the cell analyses were the following: (a) Cell Culture and High Throughput Screening (HTS) Core Facility and (b) The Biomolecule Analysis Core Facility.

\section{References and Notes}

1. Gold, K.; Cheng, Y.S.; Holmes T.D. A quantitative analysis of aerosols inside an armored vehicle perforated by a kinetic energy penetrator containing tungsten, nickel, and cobalt. Military Med. 2007, 172, 393-398.

2. Guilmette, R.A.; Parkhurst, M.A.; Miller, G.; Daxon, E.G.; Lodde, G.M.; Roszell, L.E.; Falo, G.A.; Szrom, F. Human Health Risk Assessment of Capstone Depleted Uranium Aerosols: Attachment 3 of Depleted Uranium Aerosol Doses and Risk: Summary of U.S. Assessments; Battelle Press: Columbus, OH, USA, 2006.

3. Donaldson, K.; Stone, V.; Clouter, A.; Renwick, L.; Mac Nee, W. Ultrafine Particles. Occupat. Eviron. Med. 2001, 58, 211-216.

4. Oberdörster, G. Pulmonary effects of inhaled ultrafine particles. Int. Arch. Occupat. Environ. Health 2001, 74, 1-8.

5. Pope, C.A., III; Dockery, D.W. Health effects of fine particulate air pollution: lines that connect. J. Air Waste Manag. Assoc. 2006, 56, 709-742.

6. Soto, K.F.; Carrasco, A.; Powell, T.G.; Garza, K.M.; Murr, L.E. Comparison in vitro cytotoxicity assessment of some manufactured nanoparticulate materials characterized by transmission electron microscopy. J. Nanopart. Res. 2005, 7, 145-169.

7. Murr, L.E.; Soto, K.F.; Garza, K.M. Health Hazards of Manufactured, Natural Environmental and Anthropogenic Atmospheric Nanoparticulate Materials: Past, Present and Future. In Biomaterial and Biomedical Engineering; Ahmed, W., Ali, N., Öchsner, A., Eds.; Trans Tech Publishers: Zurich, Switzerland, 2008; pp. 1-54.

8. Soto, K.F.; Garza, K.M.; Shi, Y.; Murr, L.E. Direct contact cytotoxicity arrays for filter-collected, carbonaceous (dust) nanoparticulate material and observations of lung cell response. Atmos. Environ. 2008, 42, 1970-1982. 
9. Murr, L.E. Microstructures and Nanostructures for Environmental Carbon Nanotubes and Nanoparticulate Soots. Int. J. Environ. Res. Public Health. 2008, 5, 321-336.

10. Murr, L.E.; Garza, K.M. Natural and Anthropogenic Environmental Nanoparticulates: Their Microstructural Characterization and Respiratory Health Implications. Atmos. Environ. 2009, 43, 2683-2692.

11. German, R.M. Liquid Phase Sintering; Plenum Press: New York, NY, USA, 1985; p. 251.

12. Roth, J.F.; Watchell, G.P. Heat transfer and chemical kinetics in the ignition of solid propellants. Ind. Eng. Chem. Fundamen. 1962, 1, 62-67.

13. Gold, K. Analysis of Aerosols Produced During Tests of Tungsten Alloy Kinetic Energy Penetrators; Technical Report ARWEC-TR-97014, US Army Armament Research; Development and Engineering Center: Picatinny Arsenal, NJ, USA, 1997.

14. Hetland, R.B.; Refsnes, M.; Myran, T.; Johansen, B.V.; Uthus, N.; Schwarts, P.E. Mineral and/or metal content as critical determinants of particle-induced release of IL-6 and IL-8 from A549 cells. J. Toxicol. Environ. Health A 2000, 60, 47-65.

15. Hetland, R.B.; Casser, F.R.; Refsnes, M.; Schwarts, P.E.; Lag, M.; Boere, A.F.G.; Dybing, E. Release of inflammatory cytokines, cell toxicity and apoptosis in epithelial lung cells after exposure to ambient air particles of different size fractions. Toxicol. In Vitro 2004, 18, 203-212.

16. Nelson, S.I.; Martin, T.R. Cytokines in Pulmonary Disease: Infection and Inflammation Lung Biology in Health and Disease; Marcel Dekker Inc.: New York, NY, USA, 2000.

17. Van Eeden, S.F.; Tan, W.C.; Suwa, T.; Fujji, T. Cytokines involved in systemic inflammatory response induced by exposure to particulate matter air pollutants $\left(\mathrm{PM}_{10}\right)$. Amer. J. Respir. Crit. Care Med. 2001, 164, 826-830.

18. Chung, K.F. Cytokines in chronic obstructive pulmonary disease. Environ. Respir. J. 2001, 18, 505-595.

19. Inflammatory Mechanisms in Asthma; Holgate, S.T., Busse, W.W., Eds.; Marcel Dekker Inc.: New York, NY, USA, 1998.

20. Asthma and COPD; Barnes, P.; Drazen, J.; Rennard, S.; Thomson, N., Eds.; Academic Press: New York, NY, USA, 2008.

21. Soto, K.F.; Garza, K.M.; Murr, L.E. Cytotoxic effects of aggregated nanomaterials. Acta Biomater. 2007, 3, 351-358.

22. Cugell, D.W.; Morgan, W.K.C.; Perkins, A. The respiratory Effects of Cobalt. Arch. Internal Med. 1990, 150, 177-183.

23. Clinical Environmental Health and Toxic Exposure, 2nd ed.; Sullivan, S.B., Kviezar, C.R., Eds.; Lippincott Williams \& Wilkins: Philadelphia, PA, USA, 2001.

24. Buzea C.; Pacheco, J.; Robbie, K. Nanomaterials and nanoparticles: sources and toxicity. Biointerphases 2007, 2, 17-71.

25. Murr, L.E.; Soto, K.F.; Garza, K.M. Health Hazards of Manufactured, Natural Environmental and other Anthropogenic Atmospheric Nanoparticulate Materials: Past, Present and Future. In Biomaterials and Biomedical Engineering; Ahmed, W., Ali, N., Öchsner, A., Eds.; Trans. Tech Publishing: Zurich, Switzerland, 2008; pp. 1-55.

26. Oberdörster, G.; Ferin, J.; Lehnert, B.E.; Correlation between particle size, in vivo particle persistence, and lung injury. Environ Health Perspect. 1994, 102, 173-179. 
27. Dobravolvkala, M.A.; McNeil, S.E. Immunological properties of engineered nanomaterials. Nat. Nanotechnol. 2007, 2, 469-478.

28. Sonavane, G.; Tomoda, K.; Makino. Biodistribution of Colloidal Gold Nanoparticles after Intravenous Administration: Effect of Particle Size. Colloid Surface B 2008, 66, 274-280.

29. Borm, P.J.; Robbins, D.; Haubols, S. The potential risks of nanomaterials: A review carried out for ECETOC. Part. Fibre Toxicol. 2006, 3, 11-20.

30. Shi, Y.; Murr, L.E.; Soto, K.F.; Lee, W-Y; Guerrero, P.A.; Ramirez, D.A. Characterization and comparison of speciated atmospheric carbonaceous particulates and their polycyclic aromatic hydrocarbon contents in the context of the Paso del Norte airshed along the U.S.-Mexico Border. Polycyc. Aromatic Comp. 2007, 27, 361-400.

(C) 2010 by the authors; licensee MDPI, Basel, Switzerland. This article is an open access article distributed under the terms and conditions of the Creative Commons Attribution license (http://creativecommons.org/licenses/by/3.0/). 